\title{
Why temporal networks are more controllable: Link weight variation offers superiority
}

\author{
Xin-Ya Zhang, ${ }^{1}$ Jie Sun, ${ }^{2}$ and Gang Yan $\oplus^{1,3,4, *}$ \\ ${ }^{1}$ School of Physical Science and Engineering, Tongji University, Shanghai 200092, People's Republic of China \\ ${ }^{2}$ Huawei Hong Kong Research Center, Sha Tin, Hong Kong SAR, People's Republic of China \\ ${ }^{3}$ Shanghai Research Institute of Intelligence Science and Technology, Shanghai 200092, People's Republic of China \\ ${ }^{4}$ CAS Center for Excellence in Brain Science and Intelligence Technology, Chinese Academy of Sciences, Shanghai 200031, \\ People's Republic of China
}

(Received 13 December 2020; revised 23 April 2021; accepted 3 August 2021; published 16 August 2021)

\begin{abstract}
The control of temporal networks is of paramount importance to complex systems in diverse fields. Recent studies showed that temporal networks are more controllable than their static counterparts, in terms of control time, cost, and trajectory length. However, the underlying mechanism of this intriguing phenomenon remains elusive, partly due to the fact that multiple properties of a temporal network simultaneously change over time. Here, we explore a general model of temporal networks, and prove that the weight variation of a link is equivalent to attaching a virtual driver node to that link. Consequently, the random variation of link weights can significantly increase the dimension of controllable space and remarkably reduce control cost, which unveils the fundamental mechanism for the advantages of temporal networks in controllability. The finding of this mechanism leads to a graphic criterion that allows us to further discover that, degree-heterogeneous networks are more advantageous for enhancing controllability by link weight variation, and the favorable positions of weight variation are the incoming links of the nodes with a high outdegree and a low indegree. Our results are validated in both synthetic and empirical network data, together deepening the understanding of network temporality and shedding light on the long-standing problem of establishing graphic criteria for the controllability of general dynamic systems.
\end{abstract}

DOI: 10.1103/PhysRevResearch.3.L032045

Many real-world networks are temporal, characterized by having structure or link weights that change over time in complex but meaningful ways [1-5]. Link temporality affects a wide range of dynamical processes that take place on networks, from diffusion [6-12] to random walk and search [13,14], synchronization [15-17], cooperation [18,19], and notably network controllability [20-23]. In particular, a recent study showed that temporal networks require less time, less cost, and shorter paths to be controlled than their static counterparts [22]. This phenomenon, which demonstrates the advantage of link temporality for network controllability, is interesting and counterintuitive, given that link temporality intermittently disintegrates network connectivity. Because many properties of temporal networks simultaneously change over time, such as topological structure, link weights, duration of sequential patterns, and so on, it remains unclear whether it is one of these properties or some combination of them that makes temporal networks more controllable. Furthermore, as temporal networks typically evolve under self-organizing principles, could it also be the ordering underlying such evolution dynamics that plays the determining role? Surrounded in a maze of possibilities, the important question is, what makes temporal networks special with regard to controllability?

\footnotetext{
*Corresponding author: gyan@tongji.edu.cn
}

Published by the American Physical Society under the terms of the Creative Commons Attribution 4.0 International license. Further distribution of this work must maintain attribution to the author(s) and the published article's title, journal citation, and DOI.
Here, we address this very question and prove that, regarding controllability, the weight variation of a link is equivalent to attaching a virtual driver node to that link. Topological alterations can be viewed as special cases of link weight variation, i.e., when the weight of a link changes from nonzero to zero, or vice versa. Thus, our finding implies that link weight variation is the general and fundamental mechanism for the recently discovered advantages of temporal networks in controllability [22]. Importantly, the existing criterion of temporal network controllability is algebraic and hence computationally prohibitive for large sizes. In contrast, the equivalence we have proven in the present Letter leads to a graphic criterion that maps the controllability of a temporal network to that of an effective static network. Such an equivalent mapping can significantly advance the field, because it allows us to explore the control properties of temporal networks on a large scale by using the methods previously developed only for static networks [24-38].

Nonlinear control systems are generally described as

$$
\dot{\mathbf{x}}(t)=\mathbf{f}(\mathbf{x}, \mathbf{u}, t),
$$

where $\mathbf{x}(t)=\left[x_{1}(t), x_{2}(t), \ldots, x_{N}(t)\right]^{\mathrm{T}}$ are the states of the $N$ nodes at time $t, \mathbf{f}=\left[f_{1}, f_{2}, \ldots, f_{N}\right]^{\mathrm{T}}$ are nonlinear functions describing the system dynamics, and $\mathbf{u}(t)=$ $\left[u_{1}(t), u_{2}(t), \ldots, u_{N_{\mathrm{D}}}(t)\right]^{\mathrm{T}}$ represent the external control inputs applied respectively to $N_{\mathrm{D}}$ driver nodes. The system is locally controllable along a trajectory $[\hat{\mathbf{x}}(t), \hat{\mathbf{u}}(t)]$ if its linearized system

$$
\dot{\mathbf{x}}(t)=A(t) \mathbf{x}(t)+B(t) \mathbf{u}(t)
$$


is controllable along the same trajectory $[33,34,39]$. Here, $A(t)=\partial \mathbf{f} /\left.\partial \mathbf{x}\right|_{[\hat{\mathbf{x}}(t), \hat{\mathbf{u}}(t)]}$ representing the interaction network between components (i.e., nodes), and $B(t)=\partial \mathbf{f} /\left.\partial \mathbf{u}\right|_{[\hat{\mathbf{x}}(t), \hat{\mathbf{u}}(t)]}$ denoting the specific connections between external control inputs and driver nodes. Note that matrix $A(t)$ is time varying, i.e., the interaction network between the nodes is not static but temporal. Hence, the temporality of networks plays an important role in control.

Real temporal network data are usually encoded by a sequence of snapshots [1], and the $m$ th snapshot is captured by its adjacency matrix $A_{m}$ and time duration $\tau_{m}$. As shown in a recent important advance [22], the controllable space of a temporal network with $M$ snapshots is determined by

$$
\Omega_{\mathrm{T}}=\left\langle A_{M} \mid B_{M}\right\rangle+\left(\sum_{m=1}^{M-1} \prod_{j=M}^{m+1} e^{A_{j} \tau_{j}}\left\langle A_{m} \mid B_{m}\right\rangle\right),
$$

where $\left\langle A_{m} \mid B_{m}\right\rangle=\left[B_{m}, A_{m} B_{m}, \ldots, A_{m}^{N-1} B_{m}\right]$ [see Sec. I A in the Supplemental Material (SM) [40]]. Direct calculations of Eq. (3) showed that temporal networks generally enhance controllability relative to their static counterparts [22].

However, multiple factors that might affect the controllability of a temporal network are mixed in Eq. (3), such as $A_{m}$ (the topological structure and link weights of each snapshot), $B_{m}$ (the driver node set of each snapshot), $\tau_{m}$ (the duration of each snapshot), and $M$ (the total number of snapshots). Such a mixture hinders us from exploring the fundamental question of why temporal networks are more controllable. Moreover, Eq. (3) is an algebraic criterion, which contains complicated operations of matrices, especially the matrix exponential. Hence, it is hardly applicable to evaluating the controllability of large-sized temporal networks.

Therefore, first we consider a temporal network model in which all snapshots have the same topology, the same driver node set $B$, and the same duration $\tau$. The only difference between snapshots is link weights. This model not only allows us to focus on the essential distinction between temporal and static networks, but is also general in the sense that the topological variations can be viewed as special cases of link weight variations, i.e., when the weights of links change from nonzero to zero or vice versa.

To begin with, we analyze a simple example illustrated in Figs. 1(a) and 1(b). The temporal network has two snapshots, each of which is a branching structure of three nodes. The links in the first snapshot are assigned random weights and described by a weighted adjacency matrix $A$. The links' weights in the second snapshot are the same as those in the first snapshot except for one link, from node 2 to node 3 , whose weight is perturbed from $w_{32}$ to $w_{32}+\delta$. According to Eq. (3), the controllable space of this simple temporal network is

$$
\Omega_{\mathrm{T}}=\left[B_{2}, A B_{2}, \delta B_{3}\right],
$$

where $B_{2}=[0,1,0]^{\mathrm{T}}$ and $B_{3}=[0,0,1]^{\mathrm{T}}$. If $\delta=0$, i.e., without weight variation, the network degenerates to a static counterpart [Figs. 1(d)-1(f)], whose controllable $\operatorname{dimension} \operatorname{dim}\left(\Omega_{\mathrm{S}}\right) \equiv \operatorname{rank}\left(\left\langle A \mid B_{2}\right\rangle\right)=2$ (since $A^{2}=\mathbf{0}$ here). In contrast, when $\delta \neq 0$, the network is temporal and we have $\operatorname{dim}\left(\Omega_{\mathrm{T}}\right) \equiv \operatorname{rank}\left(\Omega_{\mathrm{T}}\right)=3$, meaning that the temporal network in Fig. 1(b) is fully controllable (i.e., all three
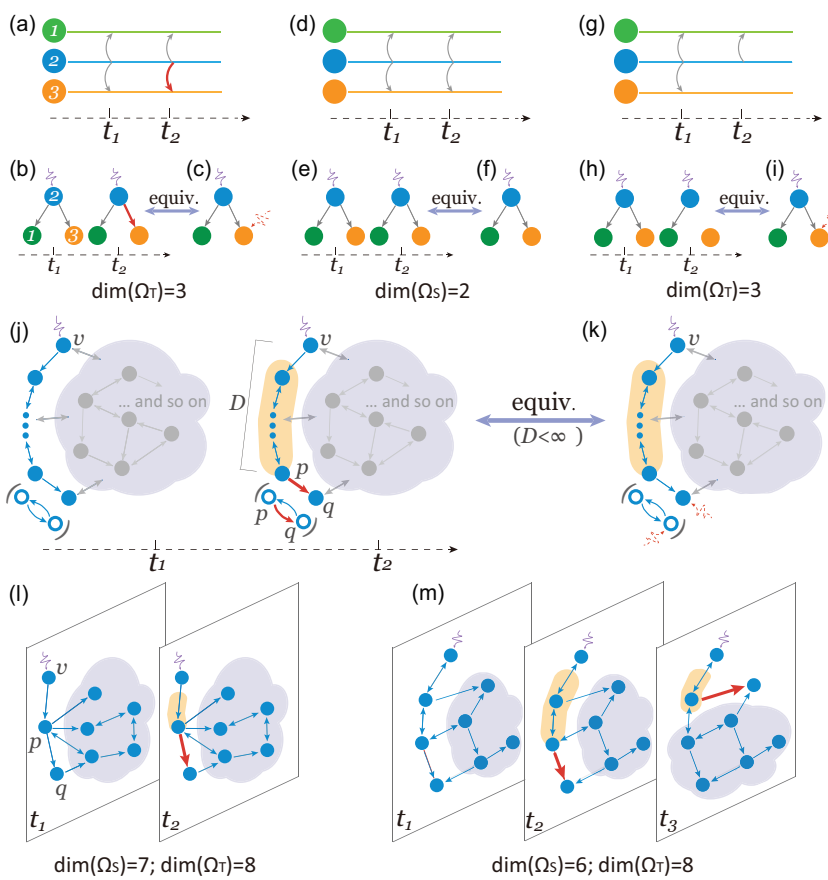

FIG. 1. Controllability of temporal networks induced by link weight variation. (a), (b) A simple temporal network with two snapshots whose topologies are the same. The only difference between the two snapshots is that the weight of a link (red, from node 2 to node 3 ) is perturbed from $w_{32}$ to $w_{32}+\delta$, where $\delta$ is a random time-invariant number. If $\delta \neq 0$, the controllable dimension of the temporal network is $\operatorname{dim}\left(\Omega_{\mathrm{T}}\right)=3$. (c) The effective static network, without weight variation but with an additional virtual driver node (pointed by the red dashed arrow), is equivalent to the temporal network (b) with regard to controllability. If $\delta=0$, the network in (a) and (b) is degenerated to a static counterpart in (d) and (e) whose controllable dimension is $\operatorname{dim}\left(\Omega_{\mathrm{S}}\right)=2$. (f) If $w_{32}+\delta=0$, i.e., the link weight is altered from nonzero to zero, the temporal network becomes topology varying $(\mathrm{g}),(\mathrm{h})$ but its controllable dimension is still $\operatorname{dim}\left(\Omega_{\mathrm{T}}\right)=3$ (i). (j) A temporal network of two snapshots with complex topologies. The weight of the link (red) from node $p$ to node $q$ is altered, and $D$ denotes the shortest path length from the real driver node $v$ to node $p$. (k) The effective static network with an additional virtual driver node, whose controllability is equal to that of the temporal network in (j). (l), (m) Two specific examples demonstrate that link weight variation alone enhances network controllability [i.e., $\left.\operatorname{dim}\left(\Omega_{\mathrm{T}}\right)>\operatorname{dim}\left(\Omega_{\mathrm{S}}\right)\right]$.

nodes are controllable). Thus, the controllable dimension is increased from 2 to 3 by the link weight variation alone.

Moreover, Eq. (4) can be recast as $\Omega_{\mathrm{T}}=\langle A \mid \hat{B}\rangle$, where $\hat{B}=\left[B_{2}, B_{3}\right]$. The recast form implies that, besides the real driver node 2 , node 3 also acts as a virtual driver node although it does not directly receive an external control input. In other words, the weight variation of a link is equivalent to attaching a virtual driver node to this link [red dashed arrow in Fig. 1(c)]. If the weight of this link is varied from nonzero to zero, the topology of the second snapshot is altered, inducing a topology-changing temporal network in Figs. 1(g) and 1(h) which also becomes fully controllable [Fig. 1(i)]. Taken together, such an equivalence has explained why temporal networks have superiority in controllability. 
Next, we prove that the equivalence we find above also holds for temporal networks with complex topologies. Figure 1(j) displays a temporal network with two snapshots, where the second snapshot $A_{2}$ is induced by the weight variation of one link in the first snapshot $A_{1}$, i.e., $A_{1}=A_{2}+\Delta$. The perturbed link is assumed to be from node $p$ to node $q$, hence matrix $\Delta$ has only one nonzero element $\delta_{q p}$ and $\Delta^{n}=0$ for $n \geqslant 2$. Let $v$ denote the real driver node, i.e., an external control input is imposed on node $v$, and $D$ denote the length of the shortest path from $v$ to $p$. Through a solid analysis (see Sec. I B in SM [40]), we show that if and only if $D<\infty$ the controllable space of the temporal network in Fig. 1(j) is determined by

$$
\Omega_{\mathrm{T}}=\left\langle A_{2} \mid \hat{B}\right\rangle,
$$

where $\hat{B}=\left[B_{v}, B_{q}\right]$. Equation (5) indicates that once there is a path from the real driver node to the perturbed link, the weight variation results in the addition of a virtual driver node (i.e., node $q$ here).

When there are multiple snapshots and the weight of a random link is perturbed on each snapshot, our extended analyses (see Sec. I C in SM [40]) show that the result of Eq. (5) can be generalized to

$$
\Omega_{\mathrm{T}}=\left\langle A_{M} \mid \tilde{B}\right\rangle,
$$

where $\tilde{B}=\left[B_{v}, B_{q_{1}}, \ldots, B_{q_{M-1}}\right]$, and $q_{i}$ with $i=1, \ldots, M-$ 1 denote the end nodes of the perturbed links. That is, although only one real driver node $v$ receives the external control input, the end nodes of the weight varied links act as virtual drivers. The result Eq. (6) has unveiled the fundamental mechanism underlying the superiority of temporal networks in controllability, which is our first main finding. This result also implies that link weight variation alone can enhance network controllability even when the topology of snapshots is invariant. Indeed, as shown in Fig. 1(1), the weight variation of one link (colored in red) is able to increase the controllable dimension from $\operatorname{dim}\left(\Omega_{\mathrm{S}}\right)=7$ to $\operatorname{dim}\left(\Omega_{\mathrm{T}}\right)=8$, although the topology does not change. Similarly, in Fig. 1(m), sequentially perturbing the weight of a link on each following snapshot leads to an increase of controllable dimension from six to eight. More numerical validations are exhibited in the SM [40].

It is worth noting that Eq. (6) has successfully mapped a temporal network into an effective static network with additional virtual driver nodes, hence the previous methods developed only for static networks, such as maximum matching [24], can be applied to analyze the controllability of temporal networks. Even more importantly, such a mapping offers us a graphic criterion which can handle temporal networks of large size. For example, in order to explore the impact of degree heterogeneity, the number of nodes needs to be relatively large. Here, we generate directed scale-free networks with a tunable exponent $\gamma$ [41] and then copy it $M-1$ times to construct $M$ snapshots. On each snapshot the weight of a random link is altered, hence the network becomes temporal by link weight variation. Its degree heterogeneity is captured by $\gamma$, i.e., smaller $\gamma$ means higher heterogeneity. The results in Fig. 2(a) show that the more heterogeneous is a temporal network, the more likely it is that the link weight variation improves controllability. Moreover, the favorable
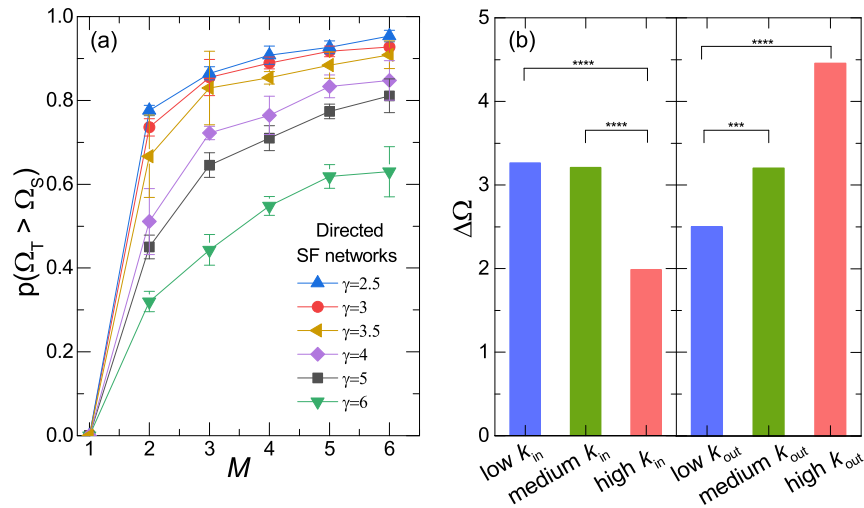

FIG. 2. Controllability enhancement vs degree heterogeneity. (a) $\mathrm{P}\left(\Omega_{\mathrm{T}}>\Omega_{\mathrm{S}}\right)$ represents the probability of that the network controllability is enhanced by link weight variation alone, where $M$ is the number of snapshots, each snapshot has 1000 nodes, and the node degrees follow a power-law distribution with exponent $\gamma$. A smaller $\gamma$ indicates higher heterogeneity. (b) The nodes are divided into three groups of equal size according to their indegrees $k_{\text {in }}$ or outdegrees $k_{\text {out }}$. The bars show the average increase of controllable dimension caused by perturbing the weights of links that point to nodes with low, medium, or high $k_{\text {in }}$ or $k_{\text {out }}$, where $\gamma=2.5$. The significance $p$ values in (b) are obtained through statistical $t$-tests, and the symbol of four asterisks indicates a $p$ value $<10^{-4}$.

positions of weight-varied links are related to the degrees of the node that the link points to. Specifically, when the outdegree of the node is high and the indegree is low, perturbing the weights of such links can achieve higher controllability [Fig. 2(b)].

When a temporal network is fully controllable, another important issue is the cost required for control. We thus explore the interesting question of whether link weight perturbation is able to reduce control cost. To proceed, we employ the widely used definition of input $\operatorname{cost} \mathcal{E}=\int_{t_{0}}^{t_{\mathrm{f}}}\|\hat{\mathbf{u}}(t)\|^{2} d t$, where
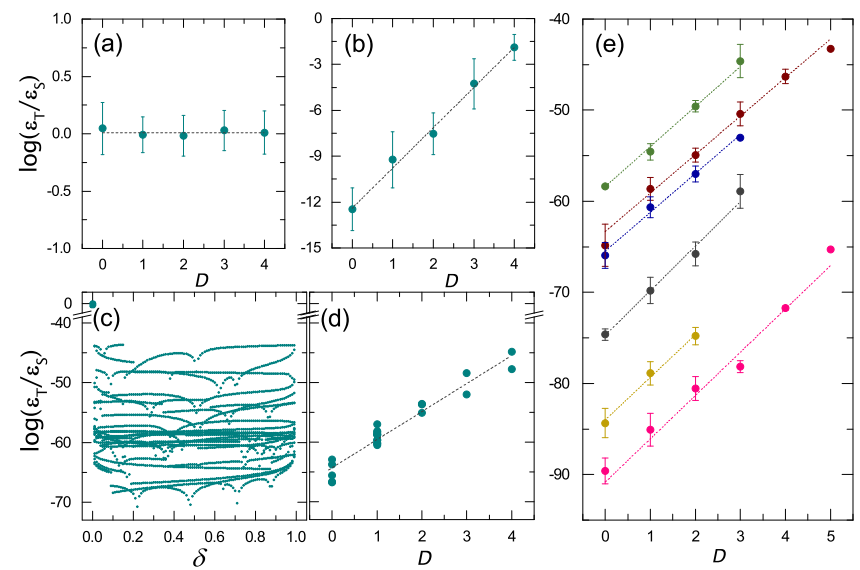

FIG. 3. Dependence of reduced control cost on distance $D$ from the driver node to the perturbed link for (a) chains, (b) circles, (d) random networks, and (e) six empirical networks (see Sec. III in SM [40]). (c) Reduced control cost vs the amount of weight perturbation for different links in a random network. The number of snapshots $M=2$, the duration of each snapshot $\tau=10^{-3}$, and the dashed lines represent best linear fits in semilog plots. 

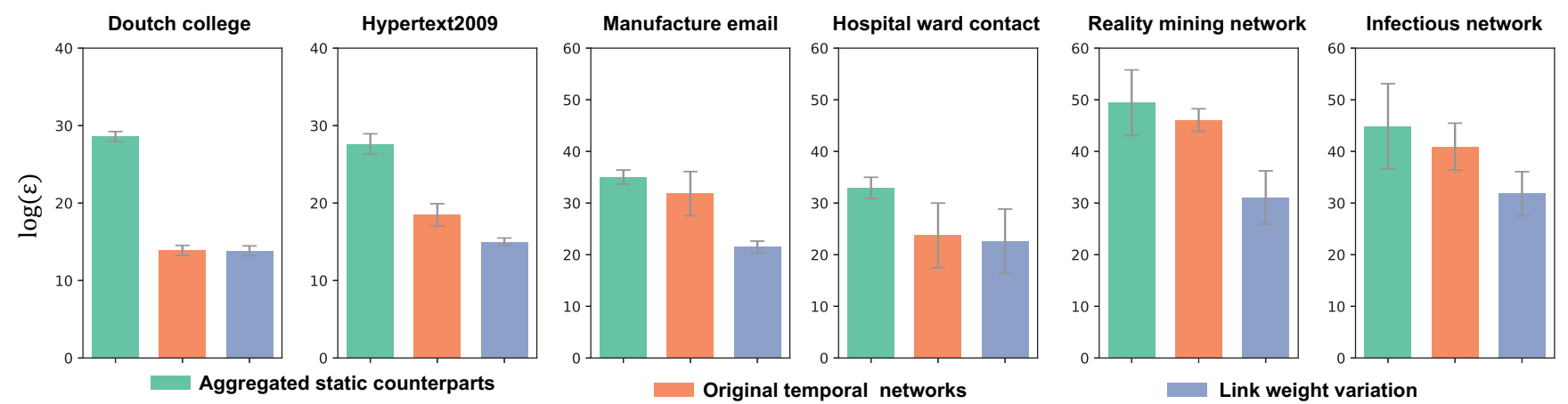

FIG. 4. Control cost of real-world temporal networks, in comparison to that induced by link weight variation and also to aggregated static counterparts. The six empirical data sets are described in SM Sec. III [40]. In each network, 20\% nodes are randomly selected as driver nodes and the snapshot duration is $\tau=10^{-2}$.

$\hat{\mathbf{u}}(t)$ is the optimal control signal [22,26,27,34] (see Sec. II A in SM [40]). Note that $\mathcal{E}$, the integral of the control signal amplitude, is a dimensionless quantity used to characterize the amount of input effort for control. Our numerical calculations show that weight perturbation, although it does not change $\mathcal{E}$ for chains [Fig. 3(a)], does reduce $\mathcal{E}$ for circles [Fig. 3(b)]. Networks usually contain both chain and circle structures, hence an arbitrary variation on the weight of a link [Fig. 3(c)] is expected to decrease control cost [Fig. 3(d)]. More importantly, the topological position of the perturbed link plays a crucial role in such an effect. Indeed, the control cost increases approximately exponentially with the distance from the driver node to the perturbed link, that is,

$$
\mathcal{E}_{\mathrm{T}} \propto e^{D},
$$

as shown in Figs. 3(b), 3(d) and 3(e) for circles, model random networks, and six fully controllable subgraphs extracted from empirical networks. Such a dependence of control cost on distance $D$ is our second main finding, which has revealed the influence pattern of link weight variation in reducing the control cost.

To further demonstrate the effectiveness of link weight variation in reducing control cost, we perform extensive tests on six empirical networks (see Sec. III in SM [40]). The static counterparts are constructed by aggregating all snapshots of an empirical temporal networks and assigning random weights to the links. To produce link weight variation, we use each static counterpart as the topology of snapshots and on each snapshot the weights of multiple random links are altered. That is, the topology is invariant but the link weights are varying; original temporal networks denote time-varying topologies in empirical data. In order to make fair comparisons we ensure that the sums of the link weights are equal for the three network types. As shown in Fig. 4, temporal networks are less costly to control than their aggregated static counterparts, which is consistent with Ref. [22]. The temporality induced by link weight variation indeed reduces control cost as well as, or in some cases even better than, the topologyvarying temporality.

In summary, our study has discovered the remarkable role of link weight variation in enhancing network controllability and reducing control cost, and unveiled the fundamental mechanism underlying the recently discovered advantage of link temporality in control. Since temporal networks not only arise in linearizations of nonlinear systems but also exist in a variety of domains, we expect that link weight variation also affects other dynamical processes upon temporal networks, especially when network asymmetry is crucial [42,43]. Moreover, our findings have bridged temporal and static network control studies, providing different opportunities for future work.

X.-Y.Z. and G.Y. acknowledge the support from National Natural Science Foundation of China (Grant No. 11875043) and Science and Technology Commission of Shanghai Municipality (Grant No. 18ZR1442000).
[1] P. Holme and J. Saramäki, Temporal networks, Phys. Rep. 519, 97 (2012).

[2] D. S. Bassett, N. F. Wymbs, M. A. Porter, P. J. Mucha, J. M. Carlson, and S. T. Grafton, Dynamic reconfiguration of human brain networks during learning, Proc. Natl. Acad. Sci. USA 108, 7641 (2011).

[3] V. Sekara, A. Stopczynski, and S. Lehmann, Fundamental structures of dynamic social networks, Proc. Natl. Acad. Sci. USA 113, 9977 (2016).

[4] D. Tang, W. Du, L. Shekhtman, Y. Wang, S. Havlin, X. Cao, and G. Yan, Predictability of real temporal networks, Natl. Sci. Rev. 7, 929 (2020).
[5] Y. Ji, W. He, S. Cheng, J. Kurths, and M. Zhan, Dynamic network characteristics of power-electronics-based power systems, Sci. Rep. 10, 9946 (2020).

[6] T. Gross, C. J. D. D’Lima, and B. Blasius, Epidemic Dynamics on an Adaptive Network, Phys. Rev. Lett. 96, 208701 (2006).

[7] N. Masuda, K. Klemm, and V. M. Eguíluz, Temporal Networks: Slowing Down Diffusion by Long Lasting Interactions, Phys. Rev. Lett. 111, 188701 (2013).

[8] I. Scholtes, N. Wider, R. Pfitzner, A. Garas, C. J. Tessone, and F. Schweitzer, Causality-driven slow-down and speed-up of diffusion in non-Markovian temporal networks, Nat. Commun. 5, 5024 (2014). 
[9] E. Valdano, L. Ferreri, C. Poletto, and V. Colizza, Analytical Computation of the Epidemic Threshold on Temporal Networks, Phys. Rev. X 5, 021005 (2015).

[10] M. De Domenico, C. Granell, M. A. Porter, and A. Arenas, The physics of spreading processes in multilayer networks, Nat. Phys. 12, 901 (2016).

[11] O. E. Williams, F. Lillo, and V. Latora, Effects of memory on spreading processes in non-Markovian temporal networks, New J. Phys. 21, 043028 (2019).

[12] A. Koher, H. H. K. Lentz, J. P. Gleeson, and P. Hövel, ContactBased Model for Epidemic Spreading on Temporal Networks, Phys. Rev. X 9, 031017 (2019).

[13] N. Perra, A. Baronchelli, D. Mocanu, B. Gonçalves, R. Pastor-Satorras, and A. Vespignani, Random Walks and Search in Time-Varying Networks, Phys. Rev. Lett. 109, 238701 (2012).

[14] M. Starnini, A. Baronchelli, A. Barrat, and R. Pastor-Satorras, Random walks on temporal networks, Phys. Rev. E 85, 056115 (2012).

[15] V. Kohar, P. Ji, A. Choudhary, S. Sinha, and J. Kurths, Synchronization in time-varying networks, Phys. Rev. E 90, 022812 (2014).

[16] X. Zhang, S. Boccaletti, S. Guan, and Z. Liu, Explosive Synchronization in Adaptive and Multilayer Networks, Phys. Rev. Lett. 114, 038701 (2015).

[17] D. Levis, I. Pagonabarraga, and A. Díaz-Guilera, Synchronization in Dynamical Networks of Locally Coupled Self-Propelled Oscillators, Phys. Rev. X 7, 011028 (2017).

[18] D. G. Rand, S. Arbesman, and N. A. Christakis, Dynamic social networks promote cooperation in experiments with humans, Proc. Natl. Acad. Sci. USA 108, 19193 (2011).

[19] A. Li, L. Zhou, Q. Su, S. P. Cornelius, Y.-Y. Liu, L. Wang, and S. A. Levin, Evolution of cooperation on temporal networks, Nat. Commun. 11, 2259 (2020).

[20] M. Pósfai and P. Hövel, Structural controllability of temporal networks, New J. Phys. 16, 123055 (2014).

[21] Y. Pan and X. Li, Structural controllability and controlling centrality of temporal networks, PLoS ONE 9, e94998 (2014).

[22] A. Li, S. P. Cornelius, Y.-Y. Liu, L. Wang, and A.-L. Barabási, The fundamental advantages of temporal networks, Science 358, 1042 (2017).

[23] Y. Zhang, A. Garas, and I. Scholtes, Higher-order models capture changes in controllability of temporal networks, J. Phys. Complex. 2, 015007 (2021).

[24] Y.-Y. Liu, J.-J. Slotine, and A.-L. Barabási, Controllability of complex networks, Nature (London) 473, 167 (2011).

[25] T. Nepusz and T. Vicsek, Controlling edge dynamics in complex networks, Nat. Phys. 8, 568 (2012).
[26] G. Yan, J. Ren, Y.-C. Lai, C.-H. Lai, and B. Li, Controlling Complex Networks: How Much Energy is Needed? Phys. Rev. Lett. 108, 218703 (2012).

[27] J. Sun and A. E. Motter, Controllability Transition and Nonlocality in Network Control, Phys. Rev. Lett. 110, 208701 (2013).

[28] S. P. Cornelius, W. L. Kath, and A. E. Motter, Realistic control of network dynamics, Nat. Commun. 4, 1942 (2013).

[29] J. Ruths and D. Ruths, Control profiles of complex networks, Science 343, 1373 (2014).

[30] J. Gao, Y.-Y. Liu, R. M. D’souza, and A.-L. Barabási, Target control of complex networks, Nat. Commun. 5, 5415 (2014).

[31] S. Gu, F. Pasqualetti, M. Cieslak, Q. K. Telesford, A. B. Yu, A. E. Kahn, J. D. Medaglia, J. M. Vettel, M. B. Miller, S. T. Grafton, and D. S. Bassett, Controllability of structural brain networks, Nat. Commun. 6, 8414 (2015).

[32] F. L. Iudice, F. Garofalo, and F. Sorrentino, Structural permeability of complex networks to control signals, Nat. Commun. 6, 8349 (2015).

[33] Y.-Y. Liu and A.-L. Barabási, Control principles of complex systems, Rev. Mod. Phys. 88, 035006 (2016).

[34] I. Klickstein, A. Shirin, and F. Sorrentino, Locally Optimal Control of Complex Networks, Phys. Rev. Lett. 119, 268301 (2017).

[35] G. Menichetti, L. Dall'Asta, and G. Bianconi, Control of multilayer networks, Sci. Rep. 6, 20706 (2016).

[36] X. Liu, L. Pan, H. E. Stanley, and J. Gao, Controllability of giant connected components in a directed network, Phys. Rev. E 95, 042318 (2017).

[37] G. Yan, P. E. Vértes, E. K. Towlson, Y. L. Chew, D. S. Walker, W. R. Schafer, and A.-L. Barabási, Network control principles predict neuron function in the caenorhabditis elegans connectome, Nature (London) 550, 519 (2017).

[38] L. Xiang, F. Chen, W. Ren, and G. Chen, Advances in network controllability, IEEE Circuits Syst. Mag. 19, 8 (2019).

[39] J.-M. Coron, Control and Nonlinearity, Mathematical Surveys and Monographs Vol. 136 (American Mathematical Society, Providence, RI, 2009).

[40] See Supplemental Material at http://link.aps.org/supplemental/ 10.1103/PhysRevResearch.3.L032045 for analytical derivation, empirical data, and more numerical results.

[41] K.-I. Goh, B. Kahng, and D. Kim, Universal behavior of load distribution in scale-free networks, Phys. Rev. Lett. 87, 278701 (2001).

[42] T. Nishikawa, J. Sun, and A. E. Motter, Sensitive Dependence of Optimal Network Dynamics on Network Structure, Phys. Rev. X 7, 041044 (2017).

[43] J. D. Hart, Y. Zhang, R. Roy, and A. E. Motter, Topological Control of Synchronization Patterns: Trading Symmetry for Stability, Phys. Rev. Lett. 122, 058301 (2019). 